\title{
Why the Length of a Quantum String Cannot Be Lorentz Contracted
}

\author{
Antonio Aurilia ${ }^{1}$ and Euro Spallucci ${ }^{2}$ \\ ${ }^{1}$ Department of Physics and Astronomy, California State Polytechnic University, Pomona, CA 91768, USA \\ ${ }^{2}$ Dipartimento di Fisica, Sezione Teorica, Università di Trieste and INFN, 34100 Trieste, Italy
}

Correspondence should be addressed to Euro Spallucci; euro@ts.infn.it

Received 6 September 2013; Accepted 18 September 2013

Academic Editor: Piero Nicolini

Copyright (C) 2013 A. Aurilia and E. Spallucci. This is an open access article distributed under the Creative Commons Attribution License, which permits unrestricted use, distribution, and reproduction in any medium, provided the original work is properly cited.

\begin{abstract}
We propose a quantum gravity-extended form of the classical length contraction law obtained in special relativity. More specifically, the framework of our discussion is the UV self-complete theory of quantum gravity. We show how our results are consistent with (i) the generalized form of the uncertainty principle (GUP), (ii) the so-called hoop-conjecture, and (iii) the intriguing notion of "classicalization" of trans-Planckian physics. We argue that there is a physical limit to the Lorentz contraction rule in the form of some minimal universal length determined by quantum gravity, say the Planck Length, or any of its current embodiments such as the string length, or the $\mathrm{TeV}$ quantum gravity length scale. In the latter case, we determine the critical boost that separates the ordinary "particle phase," characterized by the Compton wavelength, from the "black hole phase," characterized by the effective Schwarzschild radius of the colliding system.
\end{abstract}

\section{Introduction and Background}

High energy particle physics is based on the notion that smaller and smaller distance scales can be investigated by increasing the energy of the probe particle. Elementary projectiles colliding with a target can resolve distances comparable with their quantum mechanical wavelength. The more is the energy, the shorter is the wavelength in agreement with the relativistic rule of length contraction. Quantum mechanics and special relativity work together to open a window on the microscopic world.

This simple picture becomes less clear when we begin to approach the Planck scale of distance or energy and consider the concomitant quantum gravity effects. This problem has long been ignored on the basis that the Planck energy, roughly $10^{19} \mathrm{GeV}$, is so huge that no particle accelerator will ever be able to approach it.

However, the picture is completely different when we consider the string-inspired unified models with large extradimensions, where the unification scale can be as low as some $\mathrm{TeV}$. In this kind of scenario, quantum gravity effects, including microblack hole production in partonic hard scattering, have been suggested to occur near the LHC peak energy, that is, $14 \mathrm{TeV}$ [1-10]. In this new physics, the distinction between "point-like" elementary particles and "extended" quantum gravity excitations, whatever they are, that is, black holes, $D$-branes, string balls, and so forth, turns out to be fuzzy, so that standard notions, such as the Lorentz-Fitzgerald length contraction, require a substantial revision, at least insofar as its domain of validity is concerned. For instance, a fundamental, quantum string is, presumably, the smallest object in nature with a linear size given by $l_{s}=\sqrt{\alpha^{\prime}}$. Thus, in this string perspective, no distance shorter than $l_{s}$ can be given a physical meaning. Furthermore, by supplying more and more energy, higher and higher vibration modes are excited making the string longer and longer, in conflict with the length-contraction rule but not unlike the increasing size of a Schwarzschild black hole. To our mind, this signals the end of the validity of special relativity and the onset of gravitational effects.

\section{How can we account for that?}

Before trying to answer this question, it is useful to recall the derivation of the fundamental units that define the domain of quantum gravity, as the answer to our question lies in the very definition of those units. 
The appropriate standards of length, mass, and time were originally introduced by Max Planck on a purely dimensional basis by combining the speed of light $c$, the gravitational coupling constant $G_{*}\left(G_{*}\right.$ can be either the Newton constant or the higher-dimension gravitational coupling of TeV quantum gravity.), and the Planck constant $\hbar$. In other words, Planck recognized that it is possible to combine special relativity, quantum mechanics, and gravity in the following dimensional package:

$$
L_{P} \propto \sqrt{\frac{\hbar G_{*}}{c^{3}}}, \quad T_{P} \propto \sqrt{\frac{\hbar G_{*}}{c^{5}}}, \quad M_{P} \propto \sqrt{\frac{\hbar c}{G_{*}}} .
$$

Clearly, this dimensional approach defines the Planck units up to a numerical factor providing only an "orders of magnitude" estimate. In the old days, the Planck world was envisaged as the arena of violent quantum gravity fluctuations disrupting the very fabric of space and time [11, 12]. Eventually, the notion of "space-time foam" evolved into a "Planckian phase" with a different description according to string/M-theory, loop quantum gravity, noncommutative geometry, fractal space-time, and so forth.

In order to determine the numerical constants in (1), some extra argument is due.

For instance, one may declare that the Planck mass is defined by the equality between the quantum mechanical wavelength of a particle and its gravitational critical radius

$$
\frac{\hbar}{M_{*} c}=\frac{2 M_{*} G_{*}}{c^{2}}
$$

Thus,

$$
L_{*}=\sqrt{\frac{2 \hbar G_{*}}{c^{3}}}, \quad M_{*}=\sqrt{\frac{\hbar c}{2 G_{*}}} .
$$

An alternative, but consistent, definition of (3), which to our knowledge has never been noted before, is the following. $L_{*}$ is the geometric mean of the quantum mechanical wavelength $\lambda_{C}=\hbar / m c$ of the particle and its critical gravitational radius $R_{s}=2 m G_{*} / c^{2}$ :

$$
L_{*} \equiv \sqrt{\lambda_{C} R_{s}}=\sqrt{\frac{2 \hbar G_{N}}{c^{3}}} .
$$

Further insight into the physical meaning of $L_{*}$ can be obtained from the generalized uncertainty principle (GUP) [13-15], where $L_{*}$ is often identified with the string length, that is, $L_{*}=\sqrt{\alpha^{\prime}}$ :

$$
\Delta x \geq \frac{\hbar}{\Delta p}+\frac{L_{*}^{2}}{4} \frac{\Delta p}{\hbar} .
$$

By minimizing the uncertainties, one finds

$$
\Delta p_{*}=\frac{2 \hbar}{L_{*}}, \quad \Delta x_{*}=L_{*} .
$$

From (6) we see that $L_{*}$ represents the minimal uncertainty in the particle/string localization. From this point of view, $L_{*}$ is the minimal length which is physically meaningful since, for a shorter one, the uncertainty is larger than the length itself. In contrast to this, it seems worth observing that the Planck mass is neither an absolute minimum nor an absolute maximum. It is, rather, an extremal value or turning point, in the sense that, as implied by definition (2), it represents the largest mass that an elementary particle may possess or the smallest mass attributable to a microblack hole. Interestingly enough, we will argue in Section 2 as well as in Section 3 of this paper that there exists in nature a universal, unsurpassable linear energy density or tension that lies at the core of every black hole, regardless of its mass or size.

\section{Critical Boost and Minimal Length}

We have remarked earlier that the existence of a "quantum of length" $[16,17]$ is in conflict with the conventional rule of "length contraction" derived in special relativity. In a nutshell, the quantum of length $L_{*}$ is a new universal constant on the same footing as $c$ and $\hbar$, and as such it must be an observer independent. It follows that $L_{*}$ must act as an unbreakable barrier to the Lorentz-Fitzgerald contraction.

We propose to get around this problem by redefining the Lorentz-Fitzgerald contraction law in the presence of a short-distance Planck barrier. This is the crux of the following discussion.

In special relativity, a rod of length $L_{0}$ in its rest frame is seen to be contracted in the direction of motion according to the rule

$$
L(\beta)=L_{0} \sqrt{1-\beta^{2}}, \quad \beta \equiv \frac{v}{c} .
$$

An immediate consequence of (7) is that $L$ can contract to an arbitrarily small length as $\beta \rightarrow 1$. There are, however, at least two types of objections to this conclusion that require a redefinition of the contraction rule.

(i) "Quantum" objection or the absence of $\hbar$ : even though (7) is routinely applied to the world of particle physics, it was conceived with macroscopic, that is, "classical" objects in mind. Stated otherwise, the quantum of action $\hbar$ seemingly has no effect in the length-contraction rule, but we expect this to change in the ultrarelativistic regime when one approaches distances of the order of the Planck length.

(ii) "Gravitational" objection or the absence of $G_{N}$ : (7) refers to "abstract" lengths ignoring the fact that any physical object produces its own gravitational field and thus introduces a "critical" gravitational length scale, that is, the Schwarzschild radius $R_{s}=$ $2 M G_{N} / c^{2}$. If $L \leq R_{s}$, the rod is not a rod anymore, rather, it will look like a black hole! This is the so-called "hoop-conjecture": any physical object extending along a certain direction less than its Schwarzschild radius collapses into a black hole [18]. How a black hole appears in a boosted frame is an overlooked problem except in the somewhat ambiguous "shock wave limit" where $\gamma \rightarrow \infty, M \rightarrow 0$ while the product is kept finite, that is, $0<\gamma M<\infty$ [19]. 
By considering both arguments at the same time, one expects that quantum gravity imposes intrinsic limits to the relativistic contraction of physical objects. Presently, the most promising candidate for a self-consistent theory of quantum gravitational phenomena is super-string theory. From its vantage point, string theory "solves" the problem from the very beginning by assuming that the building blocks of everything are finite length, vibrating strings. Nothing can be "smaller," in the sense that any distance (length) smaller than the string length $\sqrt{\alpha^{\prime}}$ does not have physical meaning. As string theory is a quantum theory of gravity, the string length may be identified with the Planck Length $L_{P} \approx 10^{-33} \mathrm{~cm}$. Unfortunately, to our knowledge string theory says nothing about the Lorentz-Fitzgerald contraction and how to modify it.

Our foregoing discussion, on the other hand, requires that any quantum gravity-inspired extension ought to contain both Newton and Planck constant, $G_{N}$ and $\hbar$, and reproduces (7) when $G_{N}$ or $\hbar$ are "switched off".

According to the hoop-conjecture there must be a critical boost factor $\gamma_{*} \equiv 1 / \sqrt{1-\beta_{*}^{2}}$ that characterizes the transition from a gravitationally interacting two-particle system into a black hole. In order to determine $\gamma_{*}$, let us tentatively change the contraction formula into the following expression:

$$
\widetilde{L}(\beta)=L_{0} \sqrt{1-\beta^{2}}+\frac{L_{*}^{2} \theta_{H}(\beta)}{4 L_{0} \sqrt{1-\beta^{2}}},
$$

where $\theta_{H}$ is the Heaviside step function which guarantees that the extra term does not affect the measure of $L$ at rest. (We define $\theta_{H}(x)$ as $\theta_{H}(x)=1 \leftarrow x>0$ and $\theta_{H}(x)=0 \leftarrow x \leq 0$. Sometimes, it is conventionally chosen as $\theta_{H}(0) \equiv 1 / 2$. In this case, a $\beta$-independent quantity $L_{*}^{2} / 8 L_{0}$ must be subtracted in (8).) Moreover, since any macroscopic length is tens of orders of magnitude larger than $L_{*}$, the second term in (8) gives a relevant contribution only in the ultrarelativistic regime $\beta \approx$ 1. The minimum of the function $\widetilde{L}(\beta)$ is

$$
\frac{d \tilde{L}}{d \beta}=0 \longrightarrow \gamma_{*}=\frac{2 L_{0}}{L_{*}}, \quad \tilde{L}\left(\beta_{*}\right)=L_{*} .
$$

For $\gamma>\gamma_{*}$, the function $\widetilde{L}(\beta)$ "bounces back" and increases as stipulated in our earlier discussion on the basis that a similar behavior is shown by a fundamental string which cannot shrink below its minimal length $l_{s}=\sqrt{\alpha^{\prime}}$, while increasing its energy excites higher and higher vibration modes forcing the string to elongate. Thus, a natural choice for $L_{*}$ is $L_{*}=$ $l_{s}=\sqrt{\alpha^{\prime}}$. For later convenience, it seems also worth recalling again that a highly excited string looks rather like a black hole. With this identification, (9) tells that in any inertial reference frame no physical length can be smaller than the string length

$$
\widetilde{L}(\beta) \geq \sqrt{\alpha^{\prime}},
$$

and the critical boost representing the turning point between contraction and dilatation turns out to be $\gamma_{*}=2 L_{0} / \sqrt{\alpha^{\prime}}$.

Now, let us take a closer look at (8) by way of some illustrative examples. (i) Take for $L_{0}$ the Compton wavelength $\lambda_{C}=1 / m$ of a particle. In analogy with the string improved GUP, we obtain the following modified de Broglie formula:

$$
\tilde{\lambda}(\beta)=\lambda_{C} \sqrt{1-\beta^{2}}+\frac{\alpha^{\prime} \theta_{H}(\beta)}{4 \lambda_{C} \sqrt{1-\beta^{2}}} .
$$

As $\lambda(\beta)$ cannot be smaller than $\sqrt{\alpha^{\prime}}$ it follows that the mass spectrum of an "elementary" particle is bounded from above by the limiting mass $1 / \sqrt{\alpha^{\prime}}$

$$
\lambda \geq \sqrt{\alpha^{\prime}} \longrightarrow m \leq \frac{1}{\sqrt{\alpha^{\prime}}} .
$$

(ii) Next, consider the case of a Schwarzschild black hole, $L_{0}=R_{s}$. Equation (8) tells us how the horizon radius will appear from a Lorentz boosted frame as follows:

$$
R_{s}(\beta)=2 M G_{N} \sqrt{1-\beta^{2}}+\frac{\alpha^{\prime} \theta_{H}(\beta)}{8 M G_{N} \sqrt{1-\beta^{2}}} .
$$

The first term shows how the Schwarzschild radius of a moving mass appears contracted as any other physical length. The second term in (13) takes into account the existence of a "hard core" characterized by a universal, unsurpassable linear energy density or tension that prevents further contraction or collapses into a point singularity. We will come back to this essential point in the concluding section of this paper.

The critical boost is

$$
\gamma_{*}=\frac{4 M G_{N}}{\sqrt{\alpha^{\prime}}} .
$$

For $\gamma=\gamma_{*}$, the Schwarzschild radius reaches its minimal value $R_{H}\left(\beta^{*}\right)=\sqrt{\alpha^{\prime}}$. A snapshot of a black hole at this minimal size will show an object with an effective mass $M_{*}$ defined as

$$
R_{H}\left(\beta^{*}\right) \equiv 2 M_{*} G_{N} \longrightarrow M_{*}=\frac{\sqrt{\alpha^{\prime}}}{2 G_{N}} .
$$

In a string theoretical formulation of quantum gravity, the Regge slope can be related to the Newton constant through $\alpha^{\prime}=2 G_{N}$. Thus, we find $M_{*}=1 / \sqrt{2 G_{N}}=M_{P}$ and $R_{H}\left(\beta^{*}\right)=$ $L_{P}$.

If we formally assign to the black hole a Compton wavelength $\lambda_{B H} \equiv 1 / M$, we can write (13) as follows:

$$
R_{s}(\beta)=\frac{L_{P}^{2}}{\lambda_{B H}} \sqrt{1-\beta^{2}}+\frac{\lambda_{B H} \theta_{H}(\beta)}{4 \sqrt{1-\beta^{2}}} .
$$

Comparison with (11) shows that $\lambda_{B H}$ enters the modified contraction law in the inverse way with respect to $\lambda_{C}$, thus suggesting that the de Broglie wavelength of a black hole can be written as

$$
\lambda_{B H}^{d B} \equiv \frac{\lambda_{B H}}{\sqrt{1-\beta^{2}}}=\gamma \lambda_{B H}
$$


Once the critical boost $\gamma_{*}=2 M \sqrt{\alpha^{\prime}}=M / M_{P}$ is passed, the first term in (16) is negligible and the Schwarzschild radius expands as follows:

$$
R_{s}(\beta) \approx \frac{\lambda_{B H}^{d B}}{4}
$$

It is worth mentioning that, recently, a new family of singularity-free black hole-metrics was reported in [20-25], where the existence of a minimal length is assumed at the outset in the Einstein equations. A remarkable property of these black holes is to admit extremal configurations even in the neutral nonspinning case. Extremality corresponds to the lowest mass state of the system and to a minimal radius of the event horizon which equals few times the minimal length. In some simple models, it is possible to choose the free length scale that regularizes the short distance behavior in such a way that the radius of the extremal configuration is exactly the Planck length $[9,26,27]$. Without introducing the improved Lorentz law, the very idea of a minimal size object would become observer dependent.

To sum up, at this point we have

(1) equation (8) for a (semi)classical length $L_{0}$ with string corrections;

(2) equation (11) for the de Broglie wave length with string corrections;

(3) equation (13) for the Schwarzschild radius with string corrections.

Now, it is time to consider the hoop-conjecture and check the self-consistency of our formulae.

Let us start with case (1) and address the central question as follows. Can a boosted object be seen contracted below its Schwarzschild radius?

If so, the hoop-conjecture would imply the original object is seen as a black hole:

$$
\begin{aligned}
& L_{0} \sqrt{1-\beta^{2}}+\frac{\alpha^{\prime} \theta_{H}(\beta)}{4 L_{0} \sqrt{1-\beta^{2}}} \\
& \leq R_{s} \sqrt{1-\beta^{2}}+\frac{\alpha^{\prime} \theta_{H}(\beta)}{4 R_{s} \sqrt{1-\beta^{2}}} .
\end{aligned}
$$

We may regard this relation either as an equation for the radius $L_{0}$ below which the object is shielded by horizon, or as an equation for a hypothetical "terminal speed" $\tilde{\beta}$ that, once surpassed, will make the object appear inside its own Schwarzschild hoop. In the first case, it is immediate to recognize that $L_{0} \leq R_{s}$ is the $\beta$ independent, somewhat "trivial" solution. In order to be seen as a black object, the maximal length, at rest, must be smaller than the Schwarzschild radius $R_{s}$. On the other hand, if one assumes that $L_{0}>R_{s}$ and tries to determine $\widetilde{\beta}$ from (19), then one finds

$$
\widetilde{\beta}^{2}=1+\frac{\alpha^{\prime}}{4 R_{s} L_{0}}>1
$$

which is unphysical. Only by moving at a speed greater than the speed of light can an object turn into a black hole. Thus, even in the presence of quantum corrections, there is no inertial frame where a classical object with linear size $L_{0}>R_{s}$ may appear as a black hole.

Let us consider now a quantum particle rather than a classical object as follows:

$$
\begin{gathered}
\lambda_{C} \sqrt{1-\beta^{2}}+\frac{\alpha^{\prime} \theta_{H}(\beta)}{4 \lambda_{C} \sqrt{1-\beta^{2}}} \\
\leq R_{s} \sqrt{1-\beta^{2}}+\frac{\alpha^{\prime} \theta_{H}(\beta)}{4 R_{s} \sqrt{1-\beta^{2}}} .
\end{gathered}
$$

Once more, the "terminal boost" is unphysical, that is, $\beta>1$, and the only acceptable solution is

$$
\lambda_{C}=R_{s} \longrightarrow m=\frac{1}{\alpha^{\prime}}=M_{P}
$$

Thus, as before, there does not exist an inertial frame where an isolated elementary particle with (invariant) mass $m<$ $M_{P}$ looks like a black hole. However, this conclusion does not prevent the production of a black hole in the final state of a two-body high energy scattering where the hoop conjecture has been validated using numerical/computational techniques [28]. This different case will be discussed in the next section using a more analytical approach.

2.1. High Energy Collisions and Black Hole Production. We are now ready to extend (11) to the case of a two-body system of colliding partons in the framework of higher dimensional quantum gravity. In this case, the gravitational coupling constant is $G_{*}$ with dimensions (in natural units) $\left[G_{*}\right]=l^{d-1}$, much below the Planck energy, and $d$ is the number of spacelike dimensions $(\geq 3)$. If the two partons have four-momenta $p_{1}$ and $p_{2}$, it is useful to introduce the Mandelstam variable $s=-\left(p_{1}+p_{2}\right)^{2}$. In terms of $s$, we can define the "effective Schwarzschild radius" of the system as

$$
r_{H}(s)=\left(2 \sqrt{s} G_{*}\right)^{1 /(d-2)} \equiv\left(\sqrt{s} L_{*}^{d-1}\right)^{1 /(d-2)},
$$

where $L_{*}$ is the higher dimensional minimal length. The hoop-conjecture states that whenever the two partons collide with an impact parameter $b \leq r_{H}(s)$, a microblack hole is produced. In our approach, we can rephrase this statement as follows: the two-parton system will collapse into a black hole if the de Broglie wavelength (11) is smaller or equal to the Schwarzschild radius (13)

$$
\frac{1}{\sqrt{s}}+\frac{L_{*}^{2}}{4} \sqrt{s} \leq\left(\sqrt{s} L_{*}^{d-1}\right)^{1 /(d-2)}+\frac{L_{*}^{2}}{4}\left(\sqrt{s} L_{*}^{d-1}\right)^{-1 /(d-2)},
$$

where we have switched to natural units $\hbar=c=1$ and no step-function is needed as the two particles are by definition in a relative state of motion. Solving for $s$, we find the threshold invariant energy for the creation of a microblack hole. This is a necessary, but not a sufficient condition for this event to occur. 
As it can be expected, the production channel opens up once the quantum gravity energy scale is reached:

$$
\sqrt{s} \geq \frac{1}{L_{*}}=M_{*} .
$$

Equation (25) tells us that in a high energy scattering experiment we can probe distances down to $L_{*}$ but not beyond. The would be trans-Planckian region is shielded by the creation of a black hole with linear dimension increasing with $s$. This argument is the essence of a recent proposal by Dvali and coworkers $[29,30]$ to explain how quantum gravity can self-regularize in the deep ultraviolet region [26] (The scenario of UV self-complete quantum gravity is especially attractive when realized in the more general framework of $\mathrm{TeV}$ quantum gravity. In this case, the Planck scale is lowered down to the $\mathrm{TeV}$ scale, and it opens the exciting possibility to detect quantum gravity signals at LHC.). Thus, the transPlanckian regime is actually inaccessible, and the deep $U V$ region is dominated by large, "classical" field configurations. This mechanism has been dubbed "classicalization" [31, 32].

There is a second important consequence of the relation (24) regarding the final stage of black hole evaporation. Microblack holes are known to be semiclassically unstable because of Hawking radiation. However, the standard description of thermal decay breaks down when the black hole approaches the full quantum gravity regime. Even worse, no semiclassical model can foresee the endpoint of the process which remains open to largely unsubstantiated speculations.

Equation (24), on the other hand, shows not only the transition of a two-particle system into black hole, but the inverse process as well. Start from the black hole region and decrease the invariant mass of the object. Effective models of "quantum gravity-improved" black holes suggest that for $M \gg M_{*}$ the semiclassical model is correct and the particle emission is to a good degree of approximation a grey-body thermal radiation. However, as the mass of the black hole approaches the value $M_{*}$, and the size becomes comparable with $L_{*}$, the mass of the object reveals a discrete spectrum and the decay process goes on through the emission of a few quanta while jumping quantum mechanically towards the ground state. In this late stage of decay, the black hole behaves like a hadronic resonance or an unstable nucleus, rather than a hot body. Thus, it is not surprising that after crossing the critical point $M=M_{*}$ one is left with an "ordinary" elementary particle system [33].

\section{Final Remarks: A New "Black Hole Universal Constant" and the Existence of a Maximal Force in Nature}

In this note we have proposed a consistent framework to reconcile the existence of a new fundamental constant of nature, with length dimension, with the Lorentz-Fitzgerald contraction expected from special relativity. The presence of an ultimate length barrier has been related to the presence of a black hole barrier that shields the trans-Planckian regime from a direct investigation. The critical boost factor $\gamma_{*}$ that marks the sharp transition from special relativity to the "quantum gravity" regime has been related to the threshold energy where gravitationally interacting point particles collapse into an extended microblack hole. This threshold energy is determined by the final unification scale where quantum gravity becomes as strong as the other interactions.

Assuming that the "superunification" scale is the Planck scale, is there any clue as to what the expression of the "superunified force" might be? This question leads us to confront the notion of maximal tension introduced by Gibbons in the framework of general relativity [34].

Gibbons' conjecture is that there exists in nature a limiting force; let us call it a superforce, whose exact expression is given by

$$
F_{s}=\frac{c^{4}}{4 G_{N}} .
$$

With the above expression in hand, we are in a position to add some final remarks that may shed a different light on the whole sequence of arguments presented in this paper. A more comprehensive account of the following points will be presented in a forthcoming article [35].

(1) Note, first, that our definitions of Planck units are consistent with Gibbons' expression of the superforce. In other words, on dimensional grounds alone, the superforce is the "Planck force." Having established that, it takes an elementary calculation to verify that the Gibbons-Planck force $F_{s}=c^{4} / 4 G_{N}$ is, indeed, the Planckian limit of both the electrostatic Coulomb force and the static gravitational Newton's force! While this is a definite clue that (26) is the unification point of the electrogravitational force, it remains an open question whether it also represents the superunified value of all fundamental forces.

(2) With hindsight, the conspicuous absence of $\hbar$ from the Gibbons-Planck expression seems to support the classicalization idea as well as the idea of a transition from "contraction" to "dilation" in the modified expression of the Lorentz-Fitzgerald formula. Again, with hindsight, both ideas are inherent in the fundamental relationship (2)

$$
\frac{\hbar}{M_{*} c}=\frac{2 M_{*} G_{*}}{c^{2}} .
$$

As a matter of fact, inspection of the above equation shows that, on the one hand, it defines the Planck scale of mass-energy, but, on the other hand, it signals a trade-off, at the Planck scale of energy, between a quantum length (Compton) and a classical one (Schwarzschild) with the concomitant transition from "Lorentz contraction" in the particle phase to "Schwarzschild expansion" in the black hole phase.

(3) The appearance of $G_{N}$ in $F_{s}$ makes one wonder about the specific role of gravity in the unification of fundamental forces. Here we offer an alternative, gravity inspired, interpretation of the superforce: it 
represents the ultimate linear energy density of a black hole. In order to underscore this point, consider the conventional volume density of a body. In the case of a black hole, this leads to the somewhat counterintuitive result that the density is inversely proportional to the square of the mass

$$
\rho_{B H}=\frac{M}{4 \pi R_{s}^{3} / 3}=\frac{3 c^{6}}{32 \pi G_{N}^{3}} \frac{1}{M^{2}},
$$

so that, while mini black holes may possess a nuclear density, galactic black holes can be less dense than water. On the other hand, by considering the linear energy density, one obtains the universal constant

$$
\rho_{*}=\frac{M c^{2}}{2 R_{s}}=\frac{c^{4}}{4 G_{N}} .
$$

In other words, there exists in nature a limiting linear density that is a universal characteristic of all (Schwarzschild) black holes regardless of their mass or size. At first sight, this result may seem surprising and hard to understand. In actual fact, the physical explanation rests on the duality between deep UV and far IR domains in quantum gravity. The unique properties of black holes bridge the gap between trans-Planckian and classical physics [36]!

(4) Last but not least, given the background of ideas advanced in this paper, it seems natural to identify $\rho_{*}$ with the energy density of a relativistic string, and, therefore, we identify the superforce equation (29) with the universal string tension

$$
\rho_{*} \equiv \frac{\hbar c}{2 \pi \alpha^{\prime}} \equiv T_{s}
$$

Therefore, there are two equivalent ways of writing $\rho_{*}$ :

(i) classical, macroscopic form given by Gibbons' maximal tension (29);

(ii) quantum, microscopic form which is the string tension (30).

The two definitions are linked through

(i) the existence of a universal linear energy density for black holes exposing their "stringy nature." [37];

(ii) the "classicalization mechanism" of quantum gravity that identifies trans-Planckian black holes with classical, macroscopic objects.

It seems to be a unique property of gravity to bridge the gap between micro- and macroworlds.

\section{References}

[1] S. B. Giddings and S. D. Thomas, "High energy colliders as black hole factories: the end of short distance physics," Physical Review D, vol. 65, no. 5, Article ID 056010, 12 pages, 2002.
[2] M. Cavaglia, "Black hole and brane production in $\mathrm{TeV}$ gravity: a review," Journal of Modern Physics A, vol. 18, p. 1843, 2003.

[3] T. G. Rizzo, "Non-commutative inspired black holes in extra dimensions," Journal of High Energy Physics, vol. 2006, no. 9, article 21, 2006.

[4] A. Casanova and E. Spallucci, "TeV mini black hole decay at future colliders," Classical Quantum Gravity, vol. 23, no. 3, pp. R45-R62, 2006.

[5] R. Casadio and P. Nicolini, "The decay-time of noncommutative micro-black holes," Journal of High Energy Physics, vol. 2008, no. 11, article 72, 2008.

[6] D. M. Gingrich, "Non-commutative geometry inspired black holes in higher dimensions at the LHC," Journal of High Energy Physics, vol. 2010, no. 5, article 22, 2010.

[7] P. Nicolini and E. Winstanley, "Hawking emission from quantum gravity black holes," Journal of High Energy Physics, vol. 2011, no. 11, article 75, 2011

[8] M. Bleicher and P. Nicolini, "Large extra dimensions and small black holes at the LHC," Journal of Physics, vol. 237, no. 1, Article ID 012008, 2010.

[9] E. Spallucci and A. Smailagic, "Black holes production in selfcomplete quantum gravity," Physics Letters B, vol. 709, no. 3, pp. 266-269, 2012.

[10] J. Mureika, P. Nicolini, and E. Spallucci, "Could any black holes be produced at the LHC?” Physical Review D, vol. 85, no. 10, Article ID 106007, 8 pages, 2012.

[11] J. Wheeler, "On the nature of quantum geometrodynamics," Annals of Physics, vol. 2, no. 6, pp. 604-614, 1957.

[12] J. A. Wheeler and K. Ford, Geons, Black Holes, and Quantum Foam: A Life in Physics, Norton, New York, NY, USA, 1998.

[13] A. Kempf, G. Mangano, and R. B. Mann, "Hilbert space representation of the minimal length uncertainty relation," Physical Review D, vol. 52, no. 2, pp. 1108-1118, 1995.

[14] A. Kempf and G. Mangano, "Minimal length uncertainty relation and ultraviolet regularization," Physical Review D, vol. 55, no. 12, Article ID 7909, pp. 7909-7920, 1997.

[15] E. Witten, "Reections on the fate of spacetime," Physics Today, vol. 96, p. 24, 1996.

[16] L. J. Garay, "Quantum gravity and minimum length," International Journal of Modern Physics A, vol. 10, pp. 145-166, 1995.

[17] M. Sprenger, P. Nicolini, and M. Bleicher, "Physics on the smallest scales: an introduction to minimal length phenomenology," European Journal of Physics, vol. 33, no. 4, p. 853, 2012.

[18] K. S. Thorne, "Nonspherical gravitational collapse, a short review," in Magic Without Magic, J. R. Klauder, Ed., pp. 231-258, Freeman, San Francisco, Calif, USA, 1972.

[19] P. C. Aichelburg and R. U. Sexl, "On the gravitational field of a massless particle," General Relativity and Gravitation, vol. 2, no. 4, pp. 303-312, 1971.

[20] P. Nicolini, A. Smailagic, and E. Spallucci, "Non-commutative geometry inspired Schwarzschild black hole," Physics Letters B, vol. 632, no. 4, pp. 547-551, 2006.

[21] S. Ansoldi, P. Nicolini, A. Smailagic, and E. Spallucci, "Noncommutative geometry inspired charged black holes," Physics Letters B, vol. 645, no. 2-3, pp. 261-266, 2007.

[22] E. Spallucci, A. Smailagic, and P. Nicolini, "Non-commutative geometry inspired higher-dimensional charged black holes," Physics Letters B, vol. 670, no. 4-5, pp. 449-454, 2009.

[23] P. Nicolini, "Non-commutative black holes, the final appeal to quantum gravity: a review," International Journal of Modern Physics A, vol. 24, no. 7, p. 1229, 2009. 
[24] A. Smailagic and E. Spallucci, “'Kerrr' black hole: the lord of the string," Physics Letters B, vol. 688, no. 1, pp. 82-87, 2010.

[25] P. Nicolini and E. Spallucci, "Non-commutative geometryinspired dirty black holes," Classical and Quantum Gravity, vol. 27, no. 1, Article ID 015010, 2010.

[26] E. Spallucci and S. Ansoldi, "Regular black holes in UV selfcomplete quantum gravity," Physics Letters B, vol. 701, no. 4, pp. 471-474, 2011.

[27] P. Nicolini and E. Spallucci, "Holographic screens in ultraviolet self-complete quantum gravity," http://arxiv.org/abs/1210.0015 .

[28] M. W. Choptuik and F. Pretorius, "Ultrarelativistic particle collisions," Physical Review Letters, vol. 104, no. 11, Article ID 111101, 4 pages, 2010.

[29] G. Dvali and C. Gomez, "Self-completeness of einstein gravity," http://arxiv.org/abs/1005.3497vl .

[30] G. Dvali, S. Folkerts, and C. Germani, "Physics of transPlanckian gravity," Physical Review D, vol. 84, no. 2, Article ID 024039, 14 pages, 2011.

[31] G. Dvali, G. F. Giudice, C. Gomez, and A. Kehagias, "Vcompletion by classicalization," Journal of High Energy Physics, vol. 2011, no. 8, article 108, 2011.

[32] G. Dvali and D. Pirtskhalava, "Dynamics of unitarization by classicalization," Physics Letters B, vol. 699, no. 1-2, pp. 78-86, 2011.

[33] P. Meade and L. Randall, "Black holes and quantum gravity at the LHC," Journal of High Energy Physics, vol. 2008, no. 5, article 3, 2008 .

[34] G. W. Gibbons, "The maximum tension principle in general relativity," Foundations of Physics, vol. 32, no. 12, pp. 1891-1901, 2002.

[35] A. Aurilia and E. Spallucci, "Planck's uncertainty principle and the saturation of Lorentz boosts by Planckian black holes," http://arxiv.org/abs/1309.7186.

[36] B. P. Kosyakov, "Black holes: interfacing the classical and the quantum," Foundations of Physics, vol. 38, no. 7, pp. 678-694, 2008.

[37] G. T. Horowitz and J. Polchinski, "Correspondence principle for black holes and strings," Physical Review D, vol. 55, no. 10, pp. 6189-6197, 1997. 

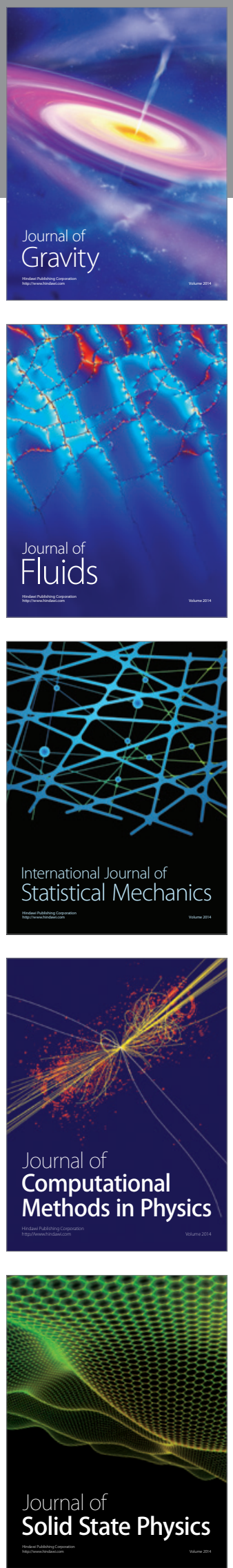

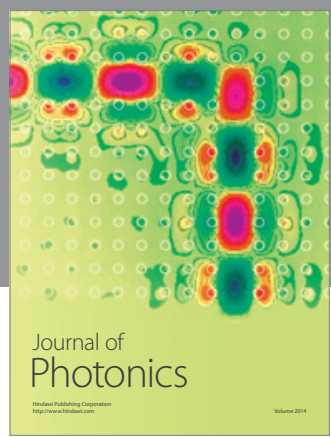

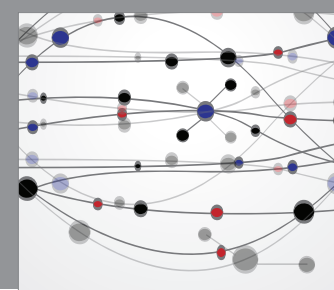

The Scientific World Journal

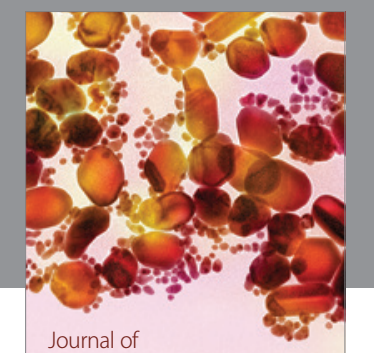

Soft Matter
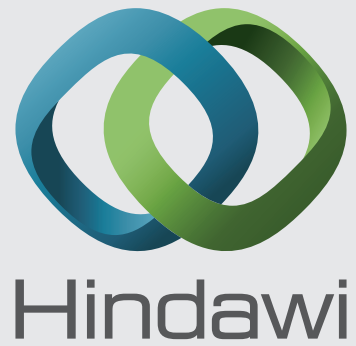

Submit your manuscripts at

http://www.hindawi.com
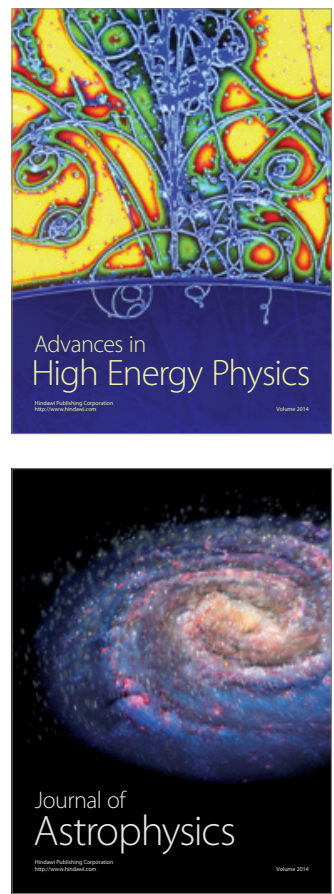
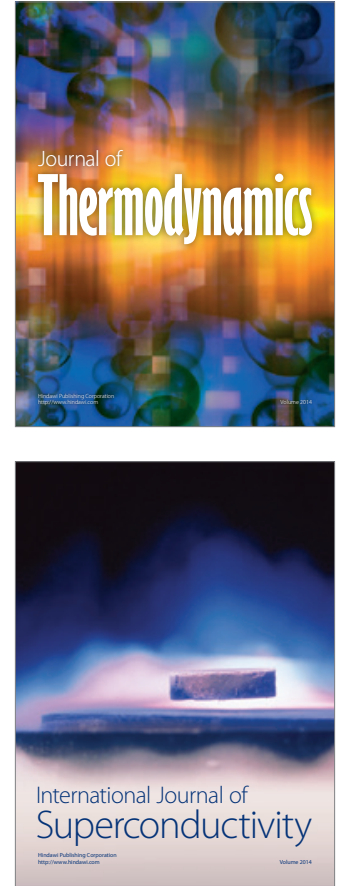
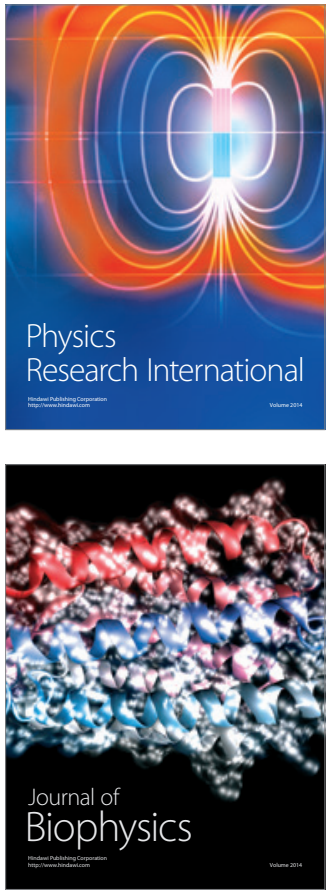
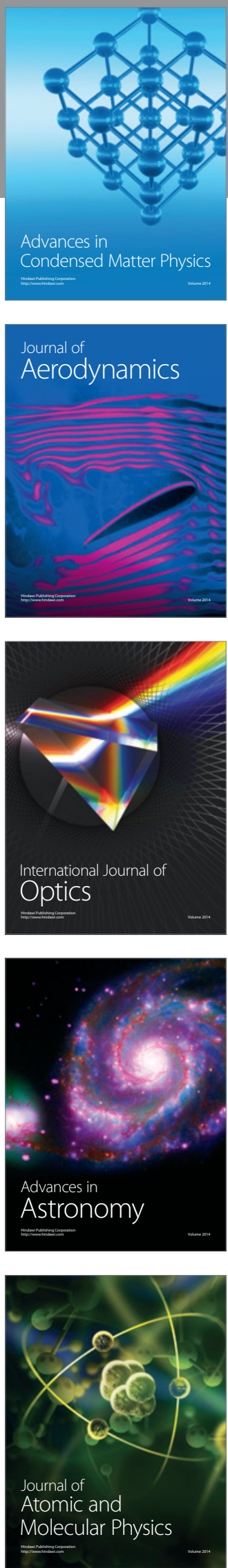